\title{
Developing a Conceptual Model to Understand the Nutritional Problems and Negative Impacts on Quality of Life among Chinese Patients with type 2 Diabetes: A Qualitative Study
}

\author{
yun wang \\ department of neurology \\ xiangping yuan \\ qianjiang district hospital \\ xianju gu \\ jiangbei district hospital \\ changxiu meng \\ rongchang district hospital \\ yanling zheng ( $\square$ yanling_swh@outlook.com ) \\ southwest hospital https://orcid.org/0000-0002-7259-2354
}

\section{Research}

Keywords: type 2 diabetes, nutrition, healthy diet, potential delay, T2DM

Posted Date: November 4th, 2020

DOl: https://doi.org/10.21203/rs.3.rs-100176/v1

License: (c) (i) This work is licensed under a Creative Commons Attribution 4.0 International License. Read Full License 


\section{Abstract}

\section{Background}

Great challenges have been reported in China with respect to the largest number of patients with type 2 diabetes mellitus and the poor nutrition management. It was generally agreed that healthy diet and regular physical activity could promote the management of diabetes, with emerging research indicating potential delay and prevention of T2DM and its complications. However, currently no conceptual model has been proposed regarding the nutritional problems and its relationship with quality of life among Chinese patients with type 2 diabetes. This study adopted a qualitative method in order to obtain a firsthand and an in-depth understanding about the nutritional problems and concerns among patients with type 2 diabetes, and explored the negative impacts of insufficient nutrition management on quality of life.

\section{Methods}

Twenty-one adult patients with type 2 diabetes from five hospitals in the southwest of China participated in a semi-structured interview regarding the nutritional problems and negative impacts on quality of life among Chinese patients with type 2 diabetes. Recordings were transcribed verbatim and content analysis were adopted to identify themes.

\section{Results}

Five themes emerged from the analysis to represent the nutritional problems and negative impacts on quality of life among Chinese patients with type 2 diabetes: knowledge about nutrition, social aspects of nutrition, cultural aspects of nutrition, behaviors and nutrition management, and health outcomes of nutrition.

\section{Conclusions}

Nutrition issues and its negative impacts on quality of life need to be considered in nursing care among Chinese patients with type 2 diabetes. Personal, social, cultural and religious factors should all be taken into consideration when promoting nutrition knowledge and behaviors.

\section{Introduction}

Diabetes has aroused global concerns by affecting 1 in 11 adults, with a total number of nearly 500 million people. As reported in 8th edition of the IDF Diabetes Atlas 2017, diabetes and its complications were responsible for four million deaths and accounted for 10 percent of global health expenditure (USD760 billion). It was estimated that 3 in 4 (79\%) of people with diabetes were living in low- and middleincome countries, which carried almost $80 \%$ of the global diabetes burden. China alone occupied the first place about the number of adults with diabetes (20-79 years) in 2017, with 121 million people suffering from diabetes. The number exceeded 40 million people more than the country of India in the second place, with a total number of 74 million. As the most prevalent form of the disease, the type 2 diabetes mellitus (T2DM) continued to rise, with over 350 million adults being currently at high risk of developing T2DM. 
Persistently high blood glucose levels in T2DM have been widely recognized to relate to a number of debilitating complications mainly affecting the heart, kidneys, nerves and eyes, which resulted in frequent hospitalizations, premature mortality and eventually reduced quality of life $\mathrm{e}^{[1]}$. It was generally agreed that healthy diet and regular physical activity could promote the management of diabetes, with emerging research indicating potential delay and prevention of T2DM and its complications ${ }^{[2,3]}$. Therefore, nutritional management combined with regular physical activity were considered as essential strategies to optimize glycaemic control and reduce long-term complications ${ }^{[4,5]}$. Adherence to these self-monitoring behaviours has been shown to improve glycaemic control, lower long-term morbidity, all-cause mortality and increased insulin sensitivity as well as decreased risk of foot ulcers ${ }^{[6-8]}$.

Although several copies of consensus reports and guidelines on diabetic nutrition management have been published in USA ${ }^{[9-13]}$, the effectiveness and benefits of nutrition care on diabetic management in China were still limited. The reason may firstly lie on the differences of dietary choices and eating habits among patients from different cultural backgrounds. For example, the people in China preferred high carbohydrate and low fat diet compared to the people in western countries who preferred high protein and fat diet such as ketogenic-diet ${ }^{[14,15]}$. This required more culturally specific and targeted nutrition advice, which eventually improved the intervention adherence. Secondly, a shortage of adequately trained diabetic specialists may reduce professional monitor on patients' nutrition behaviors and lower mutual communications ${ }^{[16]}$. Consequently, patients failed to obtain immediate feedback from healthcare workers and accordingly adjusted their nutritional plans ${ }^{[17]}$.

Therefore, this study adopted a qualitative method in order to obtain a firsthand and an in-depth understanding about the nutritional problems and concerns among patients with type 2 diabetes in the cultural context of China. Moreover, we explored the negative impacts of insufficient nutrition management on quality of life. The results of this study will assist to develop culturally-tailored and patient-centered nutritional care to promote management of glycemia, reduce complications and increase quality of life for this specific population.

\section{Methods}

\subsection{Study design and settings}

A qualitative study of semi-structured interviews was adopted to explore nutritional problems and concerns among adult patients with type 2 diabetes in the context of Chinese culture. With 56 distinctive ethnic groups, Chinese dietary culture differed a lot. Cooking styles and eating habits varied among different ethnic populations. Moreover, Chinese people preferred less meat and more veggies compared to meat lovers in western countries. Therefore, dietary and nutritional concerns among Chinese adult patients with type 2 diabetes may differ from the patients from western countries. This descriptive exploratory qualitative method allowed our researchers to collect rich narrative data and to deeply understand the feelings, thoughts and experiences of the targeted population.

\subsection{Participants}


The in-depth face-to-face interviews were performed between April and July 2020 among adult patients with type 2 diabetes who attended diabetic outpatients from five hospitals in the southwest of China. The participants were purposefully selected based on their demographic characteristics and clinical features in order to produce a heterogeneous sample. Patients attending the diabetic outpatients were identified and invited by their treating dietitian or reception nurse (both independent to the research study). The patients who met the following criteria were invited: (1) met diagnostic criteria for T2DM according to 2006 WHO report $^{[18]}$; (2) had the course of disease more than 1 year; (3) aged 18 years and older; (4) were capable of mutual communication and self-expression; (5) agreed to participate in the study. The exclusion criteria were: (1) with type 1 diabetes and gestational diabetes; (2) unable to complete the interview due to poor health; (3)with a history of mental disorders; (4) diagnosed with cognitive impairment and/or an intellectual disability.

\subsection{Data Collection}

Following a review of the literature on nutrition concerns and the impacts on health outcomes such as glycemic control and quality of life (QoL) among T2DM patients, a semi-structure interview guide was developed specifically by research group ${ }^{[19,20]}$. The guide were approved by a advisory group which consisted of senior endocrinologists, charge nurses specialized in diabetes management, and nursing experts on qualitative study. Interview questions were organized to explore participants' experience in the following aspects: (1) nutritional concerns and problems; (2) perceived barriers and facilitators affecting nutrition management; (3) the health outcomes due to nutrition management and/or nutrition problems.

After given an information sheet and verbal explanation at the start of each interview, all participants signed informed consent. The participants were reassured that whether to participate or not would not cause any impact on their treatment and care. A code name was used to protect privacy and confidentiality to ensure that each participant could not be identified through quotations and descriptions.

All interviews were conducted by our authors who were not involved in the participants' treatment and care. The interviews were organized in a waiting room to promote privacy and comfort. Semi-structured face-toface interview was conducted one-to-one at a time convenient for participants and audio-recorded.

During the interview, a variety of techniques such as communication skills, clarification probes and field notes were adopted to promote data collection. Communication skills such as reflective listening promoted participants' desires to talk and their feelings of being respected. Field notes allowed researchers to document participants' non-verbal cues but to minimize interruptions to the flow of their narratives. Clarification questions (e.g., "Could you please talk a bit more about ..." or "what did you mean by ...") were asked to encourage in-depth descriptions of participants' feelings and thoughts. Interviews varied in duration from 30 to 60 minutes. After the interview, participants were provided with a verbal summary of the interview in two weeks; and they were given opportunities to modify and clarify the interpretations.

Demographic and clinical data were collected according to patient records including age, gender, body mass index (BMI), occupation, religion, years of T2DM, family support and glycated haemoglobin levels (HbA1C).

\subsection{Data Analysis}


The interview recordings were listened repeatedly and transcribed verbatim. Each transcript was doublechecked by both interviewer and participant for accuracy. Field notes (e.g., pauses, tears in eyes, facial expressions and overall mood) were also added into transcripts. No software was used in the data analysis.

Four phases of thematic analysis approach were followed by the analysis team ${ }^{[21,22]}$. The four phases included: 『being familiar with the data by repetitive reading of interview transcripts multiple times; खgenerating initial codes from transcripts by identifying interesting, convergent and divergent features within

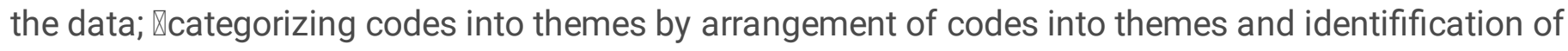
relationships between the different codes; and 『reviewing and cross-checking the themes. Both of bottom up (inductive) and top-down (theoretical) approaches were adopted when generating themes. An inductive reasoning allowed the codes and themes to emerge through repetitive review of the transcripts, while a deductive reasoning allowed a further examination of the themes in relation to the interview guide and the existing findings.

For bottom-up analysis, after the first four interviews, an initial codebook was developed by the research team. And then two of the authors W.Y. and Z.Y. independently coded the remaining transcripts, and added additional codes as appropriate. The interpretation of the codes were then discussed and the themes were chosen by these two researchers until agreement was reached based on consensus negotiation. In the final stage, in a top-down manner, themes were redefined and additional themes were developed, with comparisons to previous findings in the literature and our interview guide; and to ensure adequate coverage of all the specific topics this study addressed. To improve the rigour of the findings, themes were crosschecked and agreed by all authors. The authors have different educational backgrounds (diabetes, nutrition, nursing, sociology).

Data saturation was set at the point in which no new data emerged. Saturation of themes was reached prior to the completion of 19 interviews; however, two more interviews were conducted ensure a diverse sample considering participants' demographic characteristics and disease features.

\subsection{Ethical approval}

Ethical approval was obtained from medical ethics committee in hospitals. All participants provided informed written consent. We conducted this study in accordance with The Code of Ethics of the World Medical Association (Declaration of Helsinki).

\section{Results}

Table 1 presented the demographic and clinical features of the interview participants. Their age ranged from 45 to 77 years old, with a mean of 61.52 (SD: 10.81). The sample included 11 males (52.4\%) and 10 females (47.6\%). The average years of suffering from T2DM was 10.27 (SD: 5.07). Hemoglobin A1c (HbA1c) was $9.02 \pm 4.42 \%$ on average. 
Table 1

Characteristics of the participants

\begin{tabular}{|c|c|c|c|c|c|c|c|c|}
\hline Participant & Gender & $\begin{array}{l}\text { Age } \\
\text { (Years) }\end{array}$ & $\begin{array}{l}\text { BMI } \\
\left(\mathrm{kg} / \mathrm{m}^{2}\right)\end{array}$ & Occupation & Religion & $\begin{array}{l}\text { Years } \\
\text { of } \\
\text { T2DM }\end{array}$ & $\begin{array}{l}\text { Living } \\
\text { with }\end{array}$ & $\begin{array}{l}\text { HbA1C } \\
(\%)\end{array}$ \\
\hline 1 & Female & 73 & 29.86 & Retired & No & 10 & Family & 8.4 \\
\hline 2 & Male & 67 & 23.51 & Home & Islam & 11 & Family & 5.8 \\
\hline 3 & Male & 47 & 26.2 & Staff & No & 5 & Wife & 7.8 \\
\hline 4 & Male & 79 & 24.22 & Retired & No & 8 & Wife & 6.3 \\
\hline 5 & Male & 46 & 22.5 & Freelance & No & 9 & Alone & 6.5 \\
\hline 6 & Male & 60 & 28.14 & Resident & No & 19 & Alone & 9.8 \\
\hline 7 & Female & 77 & 21.36 & Retired & No & 20 & Family & 9.2 \\
\hline 8 & Female & 55 & 17.9 & $\begin{array}{l}\text { Public } \\
\text { officials }\end{array}$ & No & 10 & Husband & 11.5 \\
\hline 9 & Female & 71 & 29.07 & $\begin{array}{l}\text { Public } \\
\text { officials }\end{array}$ & No & 11 & Family & 8.1 \\
\hline 10 & Male & 50 & 17.91 & Resident & Christian & 1.5 & Wife & 7 \\
\hline 11 & Female & 72 & 34.85 & Retired & No & 7.2 & Husband & 6.7 \\
\hline 12 & Male & 67 & 26.25 & Retired & No & 13 & Alone & 9.6 \\
\hline 13 & Male & 52 & 24.5 & Resident & Buddhism & 10 & Family & 11.5 \\
\hline 14 & Female & 73 & 21.36 & Retired & No & 20 & Husband & 9.3 \\
\hline 15 & Male & 64 & 16.89 & Farmer & Buddhism & 13 & Wife & 8.7 \\
\hline 16 & Female & 66 & 22.2 & Retired & No & 6 & Alone & 7.2 \\
\hline 17 & Male & 67 & 24.64 & Retired & Buddhism & 10 & Wife & 8.1 \\
\hline 18 & Male & 53 & 24.80 & $\begin{array}{l}\text { Public } \\
\text { officials }\end{array}$ & No & 6 & Wife & 5.3 \\
\hline 19 & Female & 45 & 30.00 & $\begin{array}{l}\text { Public } \\
\text { officials }\end{array}$ & Buddhism & 2 & Alone & 14.1 \\
\hline 20 & Female & 58 & 16.65 & Home & No & 6 & Family & 3.3 \\
\hline 21 & Female & 50 & 23.24 & Home & No & 3 & Husband & 25.3 \\
\hline
\end{tabular}

Five themes emerged from the analysis to represent the nutritional problems and negative impacts on quality of life among Chinese patients with type 2 diabetes: knowledge about nutrition, social aspects of nutrition, cultural aspects of nutrition, behaviors and nutrition management, and health outcomes of nutrition. 


\section{Theme 1: Knowledge About Nutrition}

Almost a half of the respondents (number=10) complained of the insufficient knowledge of nutritional education. Some doctors and nurses may have been fully occupied with therapies and operations, they were too busy to provide nutrition knowledge.

"The healthcare workers only gave me insulin pump and said nothing more. There were no doctors or nurses telling me about how to control my diet, not mention to discussing with me about what to eat and what not to eat in details."(P12)

Although they have been educated by healthcare workers, five patients considered that the nutritional knowledge they were given were too broad and too superficial. They still expressed difficulties with the application of these information into daily meals because they did not know how to accurately control diet and precisely calculate calories.

"The doctor only said that 'the diet should be light; the amount of rice and noodles should be reduced; and more vegetables should be eaten'. That's all the doctor said. However, I am still confused about how much exactly I should reduce the amount of rice and noodles. Their nutritional advice is helpless, and I still have to learn by oneself through reading a guide book, or looking up on the Internet."(P8)

"I have no idea of what high-quality protein is. Tofu? seafood? There are thousands of kinds of food. I am really confused of what we could eat and what we could not."(P21)

One-third of the participants (number=7) mentioned about the over-theorized knowledge. According to the traditional algorithm and theory provided by health-care workers, the amount and frequency of food intake was complex and difficult to calculate.

"As the doctors and nurses said, according to guidelines and theories on diabetes, my meals were required to be carefully controlled through calculating the precise amount of overall food intake. That means, you need to understand the calories of each kind of food, and also you need to weigh the precise amount of the food, and then you need to make some calculations. These process are beyond my understanding as an old guy. I just quit."(P04)

Moreover, inaccurate knowledge were mentioned by 11 participants. There were some misunderstandings and wrong perceptions on diabetic nutrition. For example, some diabetics even refused to eat any kind of fruit.

"Diabetic patients can not eat any fruit because all fruits contain sugar. One of my friends, who has had diabetes for over 10 years, told me to cut back on any sweet, especially fruit. We believe that blood sugar would rise immediately after eating fruits and vegetables."(P01)

"I had an old friend who had retired and raised bees, and he brought me two big bottles of bee honey. He told me that honey was not high in sugar and was good for health with no side effects to diabetes. Therefore I 
ate rice gruel and steamed bread with a spoonful of honey everyday. But as a result, my blood sugar went up high immediately."(P03)

\section{Theme 2: Social Aspects of Nutrition}

Most of patients with type 2 diabetes were living with family members; therefor it was generally recognized that family support was a key to successful diabetic self-care. Foods acted as an important bridge connecting family members; therefore, patients' nutrition habits were greatly impacted by their family members. Six respondents (28.6\%) discussed that they would like to sacrifice themselves for their family. In other words, they gave priority to the nutritional needs of children or other family members over their own. If there was a conflict of the therapeutic diet with the family's dietary preferences, the patients would go against the diabetic diet and consider their family's eating habits.

"I am a diabetic patient, but at the same time, I am also a mother. If I reduce the amount of the oil and sugar too much when cooking, my son won't eat the food because it is not tasty. Besides, my son needs enough energy for growing up. I could not require him to eat diabetic diet along with me. That is not fair for him. Therefore I cook and eat normal foods with my son; I rely on medicine to help me control the glucose."(P19)

Three patients $(14.3 \%)$ recommended that their family members also need to command essential knowledge on diabetic nutrition. If the family members lack relevant knowledge, they may fail to support patient's diet control.

"My son is very kind to me. He always bought me a full box of fruit. There was a time that he sent me a lot of mangoes by Federal Express. The mango was so big in size, I could only ate a half. But it is wasteful to throw away the rest of the mango. So I ate the whole mango. And I still have a full box of mangoes waiting for me to eat up. I told my son not to buy so many fruits because I have diabetes; but he still because he loves me."(P11)

Over one third of the participants (number=8) discussed about the stress came from multiple social roles may affect diet management and blood sugar control. Patients often ignored their own diet needs because of their responsibilities to family and work. For example, female diabetics were busy with family care while working at the same time, so they had no time to take care of their own diet control. For male diabetic patients, they complained more pressure from work and social interactions, so they chose to give up diabetic diet.

"I knew I had diabetes, but I was too busy at work to prepare myself a diabetic meal. Moreover, my child was at a key time for the preparation of college entrance examination, I spent my rest of time to take care of him. I just ate whatever I could have."(P19)

"Before when I was working as a manager, I did have diabetes. But at that time, I had lots of social interactions, with my colleagues, with my clients and with my working partners. We gathered together to eat and drink. Every time I drank a lot alcohol, at least five to six bottles."(P03) 
"I often discuss business and eat outside, and sometimes I even have to drink until the middle of the night. I know I can't control my diabetes if I eat like this, but I have to work to earn money".(P05)

\section{Theme 3: Cultural Aspects of Nutrition}

Given that the Chinese culture generally associated with collectivism and hospitality, it was impolite to refuse drinking and eating during social gatherings with family and friends. Some patients even worried about stigmatization as a result of their conditions. This perception usually affected these patients' adherence to the nutritional recommendations.

"When I went to a dinner party, all my friends were eating and drinking. I didn't want to look like antisocial to them. Besides, I didn't want them to know I had diabetes. I just ate as the same way as they did." (P5)

In addition, religions were important factors that influencing nutritional habits. Two participants reported themselves as a Islam religion. They mentioned about the conflicts between their religion culture and diet habit.

"In our religion we have a whole month of Ramadan (a festival of Fast-Breaking). Even as a diabetic patient, we still follow our religion culture and abstain from food and drink during daylight hours in Ramadan. There were several times I got dehydration and hypoglycemia during Ramadan."(P13)

"I only ate meals before sunrise and after sunset. I did not eat anything, and even medicine at daytime. After Ramadan, we have Corban Festival. We slaughter sheep and cattle, and eat our favorite traditional foods to celebrate. Although this will cause unstable blood sugar, but we still follow the traditional customs."(P17)

\section{Theme 4: Behaviors and Nutrition management}

\subsection{Hard to follow dietary recommendations}

The patient's behaviors of diet control goes to two extremes, either excessive restriction of food, or no restraints.

"I knew diabetic diet was important and essential to glucose control. But I was born to love meat and desserts. I would not fell happy if I quit them. I could not completely change my diet habits. So I just ate whatever I wanted. If the blood glucose got fluctuated, I just ate one more pill."(P4)

"It is heard that eating fish and meat is easy to raise blood sugar. So I do not eat any fresh meat, even eggs; I only eat vegetables. This time when I was resident in hospital, my doctors said that my protein index was low. I assumed that low protein index was related to my strict restriction on meat."(P08)

\subsection{Unfixed timetable of food intake}


Three interviewees complained that they felt it difficult to strictly adhere to meal plans due to changes on life styles or work schedules. Moreover, they did not know how to adjust their diet according to the changes.

"I work in a police station. Sometimes I fail to eat my meals on time when there is an accident or an emergency. I have to deal with the case before I could eat my meal. The doctors' dietary advice were only suitable for patients living in a regular schedule. It did not suit people like me whose work schedule was not fixed."(P18)

Although some diabetic patients realized the importance and benefits of a balanced diet to glucose control, seven participants still found themselves hard to comply with a diabetic diet. Some type 2 diabetics refuse to have low fruit and vegetable intake simply because they don't like it. In addition, some patients do not like to eat vegetables because they feel that the taste is light and does not conform to their personal taste preference.

"My husband and I both have diabetes. I adhere to dietary requirements very well, but my husband is obstinate and self-willed. Sometimes he sneaked out to eat ice-cream and high-sugar foods. Then he got blood glucose fluctuations."(P7)

\subsection{Nutrition information seeking}

Diabetics reported high demands of nutrition information since the information seeking behaviors was evident in most participants $(15,71.4 \%)$. Respondents discussed a variety of ways they looked for and accessed information related to nutrition, including surfing on internet, talking with other patients, attending health professional lectures, and reading newspaper and cookbook.

"The doctors are so busy that they only tell you briefly and roughly about how to eat. As a patient, we still need to search for and learn about nutrition knowledge on our own, such as reading, online surfing."(P21)

However, none of the respondents reported an effective source that was convenient to seek information to control diabetic diet. One reason was that some nutrition information was too professional to understand for patients. Moreover, complaints about the quality of online information were also raised. For example, information on internet were over-loaded and contradictory, it was difficult for patients to distinguish between true and false.Third, they also worried about the leak of personal information while log into online education platforms.

"I attended a lot of lecturers on nutrition organized by hospitals and communities; and I did take notes carefully every time. But my memory was poor. I could not remember exactly how to eat. It was troublesome and inconvenient to glance over all my notes. So I just gave up the notes and simply ate as the same as before."(P15)

"The online information and education was convenient, but we still doubted the reliability of these information. I heard that personal information was leaked and money were cheated on the internet. So I did not think the network was still not very safe."(P20) 


\section{Theme 5: Health outcomes of Nutrition}

\subsection{Psychological stress}

Eleven interviewees reported feelings of high alerts due to worries and fear of hypoglycemia. They chose to eat extra meals if they feel slightly uncomfortable in case of hypoglycemia. Sometimes they even completely ignored diet control to avoid hypoglycemia.

"If I have a feeling of empty in my stomach, I will have some food as soon as possible. I am not sure whether it is hypoglycemia or not. But I still have something to eat in order to prevent it (hypoglycemia)." (P15)

"When my blood sugar is low, I feel weak and uncomfortable. On the contrary, when my blood sugar is high, I don't feel anything. Therefore I don't pay much attention to my diet when my blood sugar is high." (P20)

Diabetes was a chronic and lifelong disease. Long-term self-management and worries about complications caused huge psychological pressure and mental burden to patients. Five participants expressed their lack of confidence and low self-efficacy on lifelong blood glucose control. This may first because they do not command enough and correct knowledge on nutritional diet, so they have no idea of how to correctly control blood glucose; and another reason may lie in that the fluctuations of blood sugar do not bring them positive feedback and sense of benefits.

"Through diet control, I could never command the change patterns of blood sugar, which is always high or always low. I am too tired and bored to monitor the diet and the glucose . I want to give up."(P21)

"I have been too anxious to sit down since the diagnosis of diabetes. I am angry with myself because my parents and sisters do not have diabetes. I do not know how I can get this disease. I feel overwhelmed, and I even doubted that it was a punishment from Gods."(P10)

"When my blood sugar is unstable, I often feel upset and particularly sad. Why it was me who got the disease, and why I could not eat sweet food and eat whatever I want, just like my other friends have done."(P06)

\subsection{Poor glucose control}

One of the evident impacts of diet is the fluctuant blood sugar. Failing to follow strict diet management may result in poor glucose control.

"When my blood sugar went up a little high, my daughter asked me to control my diet first, saying that 'if I didn't control my diet well, I would soon get diabetes'. I didn't believe it at all, and I still ate dates all day long. As a result, after half a year, my fasting blood sugar reached more than 10."(P14) 


\subsection{Diabetic complications}

The fluctuant blood sugar caused by uncontrolled diet management may also lead to a variety of diabetesrelated complications, such as diabetic nephropathy, diabetic retinopathy, cardiovascular and cerebrovascular diseases and so on. The complications increased the physical and economic burdens to patients, which were closely associated with the decrease of health quality of life.

"Actually I knew my blood sugar was high five years ago, but I didn't take it seriously. I still ate whatever I wanted. Anyway, it didn't hurt or itch, until I got some problems on my eyes. I regretted that I did not listen to the doctor's advice on diet control at the beginning."(P06)

\subsection{Reduced quality of life}

The experience of daily insulin injection and the fear of hypoglycemia and diabetic complications placed a negative impact on daily activities and social interactions, eventually resulting in a decrease on quality of life. Most of the respondents suffered reduced life quality when they were too cautious about low blood glucose. They have had to prepare candy and nuts in case of hypoglycemia everywhere they went, including at home, at workplace and during traveling.

"I can not accept that I have to get insulin injection before eating every day; and also I can not bear that I have to take my insulin pen when going out for a long distance. All of these are inconvenient."(P12)

"There is a joke saying that 'it is all right to go out without taking money, but it is bad to go out without candy'. I know the bad feeling of hypoglycemia, so now I have sugar all over my house. If I don't have a few sweets handy, I'm in a panic."(P09)

Moreover, some participants even mentioned behaviors of avoidance and restrictions on physical exercises and social interactions. The interviewees were overly cautious about hypoglycemia so that they decreased the number of going out and reduced the length of social gathering. One respondent even gave up driving due to the potential risks of hypoglycemia.

"I do not dare to go far. I only take a walk around my house; therefore I could go back to home in a short time if I feel uncomfortable."(P04)

"Generally, I seldom go out and travel around, because it is very inconvenient that I have to prepare medicine and insulin. Besides, I keep worrying about emergency situations such as hypoglycemia."(P07)

"I quit driving a car on my own. When I have a hypoglycemia attack, I shiver and can not move my body. I am afraid this will cause me into danger if I am driving. (P08)

\section{Discussion}

Most of our interview participants recognized that dietary control helped them manage their diabetes, and also obtain the psychological and physical benefits. The lack and inaccuracy of knowledge about diabetes 
management was still prevalent among Chinese patients with T2DM, although health-care professionals have been made great efforts to promote nutrition education among diabetic patients. Three issues were mentioned about nutrition education: insufficient, inaccurate, and over-theorized (Fig. 1). More specifically, participants' complaints were: limited time to communicate with health-care workers, limited accessible to nutrition knowledge, and limited understanding of complicated terminology and calculations. However, conflicts raised considering the medical and referral systems in China. Patients were able to visit tertiary hospital with freedom and without any appointment. Therefore, Chinese diabetic specialists also complained that their great workloads in clinical settings did not allow them to spend enough time to provide detailed and precise knowledge and information even though they wish to do so. These results highlighted the needs of effective interventions on information provision and training during diabetic care. A multidisciplinary team (MDT) including nurse specialists and nutritionists may be a potential solution to promote nutrition education and management. Another feasible solution was to adopt e-Health technologies and develop mobile Apps to assist nutrition management ${ }^{[23]}$. Electronic health (eHealth) and mobile health (mhealth) technologies such as web-based programs and smartphone applications, have demonstrated as effective in assisting individuals with diabetes self-management (DSM) behaviors ${ }^{[24]}$. For instance, Dr. Denecke and colleagues ${ }^{[25]}$ have developed and implemented the mobile app GlucoMan to support data collection, allowing patients to monitor disease-relevant parameters; however, more functions need to be developed regarding nutrition care and dietary management. Just as Dr. Gifford and the research team reported in their qualitative investigation, the identified domains to improve nutrition literacy included: specific nutrients versus foods, labels and packaging, construction of the diet, knowledge and guide to healthy eating, understanding of serve and portion sizes, ability to select healthier foods, and demographics such as belief systems and culture ${ }^{[26]}$.

As shown in Figure 1, the consequences of knowledge inaccuracy and insufficiency were misunderstanding and negative attitudes towards nutrition management and eventually harming behaviors such as adherence to medical treatment. Most of current interventions were focused on how to improve relevant knowledge. Nevertheless, according to the knowledge, attitude, and practice (KAP) model ${ }^{[27]}$, changes on nutrition behaviors went through three continuous processes: acquiring knowledge, producing beliefs, and forming behaviors $^{[28]}$. This reminded diabetic care workers of that both of cognitive factors (knowledge) and emotional factors (attitudes and perceptions) should be considered to promote dietary behaviors.

Most importantly, same as previously reported results ${ }^{[29,30]}$, the patients themselves brought up the risks of hypoglycaemia as a major concern. Hypoglycaemia was one of most complained acute complications in diabetes, which may threaten patients' lives. In addition, as our participants reported, hypoglycaemia has also been positively associated with increased rates of mental disorders such as anxiety and depression, with increased deficits on working capabilities, and with reduced level of social interactions. All of these will eventually reduce patients reported outcomes (PROM) including quality of life (Fig. 1).

Personal, social, cultural and religious factors should all be taken into consideration when promoting nutrition management among Chinese patients ${ }^{[31]}$. In this study, we explored factors that may impact nutrition management according to the bioecological model developed by Urie Bronfenbrenner ${ }^{[32]}$. Micro- 
system included the factors that were most closely related to individual development and health.

Demographics, nutrition knowledge and disease features were identified in this study. Meso-system referred to the mutual connections between the factors in microsystems. The micro-systems with strong positive connection could produce the optimization effect of $1+1>2$; otherwise, it may have a negative effect on individuals. For example, if an inpatient could get support from the family system, the patient could adjust to the ward environment quickly and more adhere to the treatment. According to our findings and participants' perspectives, family, working community, and social interactions were included in Meso-system. Macrosystem were factors that with the furthest relationship with individuals but the most extensive scope, such as culture, religion, community culture reported in our results.

\section{Conclusion}

This study identified key issues regarding nutrition self-care specific to patients with type 2 diabetes under the Chinese context. Personal, social, cultural and religious factors were also identified within the context of ecological model. A conceptual model were then developed to illustrate the nutrition problem and its negative impact on quality of life. Recommendations were given that innovative and effective interventions to bridge mutual communications on nutrition self-care between diabetic specialists and diabetics.

\section{Abbreviations}

T2DM: type 2 diabetes mellitus

BMl: body mass index

HbA1C: glycated haemoglobin levels

QoL: quality of life

PROM: patients reported outcomes

MDT: multidisciplinary team

KAP: knowledge attitude and practice

DSM: diabetes self-management

\section{Declarations}

\section{Ethics approval and consent to participate}

Ethical approval was obtained from the medical ethics committee within the hospitals.

\section{Consent for publication}




\section{Availability of data and material}

The datasets used and/or analysed during the current study are available from the corresponding author on reasonable request.

\section{Competing interests}

None.

\section{Authors' contributions}

Study design: $Y W, C M$ and $Y Z$. Data collection: $Y W, X Y, X G, C M$ and $Y Z$. Data analysis: $Y W, X Y$ and $Y Z$. Data interpretation: $Y W, X Y, X G, C M$ and $Y Z$. Manuscript draft: $Y W$. Manuscript revision: $C M$ and $Y Z$. Manuscript approval: $Y W, X Y, X G, C M$ and $Y Z$.

\section{References}

1. Gregg EW, Sattar N, Ali MK. The changing face of diabetes complications. Lancet Diabetes Endocrinol. 2016. 4(6): $537-47$.

2. Ley SH, Hamdy O, Mohan V, Hu FB. Prevention and management of type 2 diabetes: dietary components and nutritional strategies. Lancet. 2014. 383(9933): 1999-2007.

3. Evert AB, Boucher JL, Cypress M, et al Nutrition therapy recommendations for the management of adults with diabetes. Diabetes Care. 2013. 36(11): 3821-42.

4. Guo Y, Huang Z, Sang D, Gao Q, Li Q. The Role of Nutrition in the Prevention and Intervention of Type 2 Diabetes. Front Bioeng Biotechnol. 2020. 8: 575442.

5. Mijatovic-Vukas J, Capling L, Cheng S, et al Associations of Diet and Physical Activity with Risk for Gestational Diabetes Mellitus: A Systematic Review and Meta-Analysis. Nutrients. 2018. 10(6).

6. Young LA, Buse JB, Weaver MA, et al Glucose Self-monitoring in Non-Insulin-Treated Patients With Type 2 Diabetes in Primary Care Settings: A Randomized Trial. JAMA Intern Med. 2017. 177(7): 920-929.

7. Chrvala CA, Sherr D, Lipman RD. Diabetes self-management education for adults with type 2 diabetes mellitus: A systematic review of the effect on glycemic control. Patient Educ Couns. 2016. 99(6): 926 43.

8. He X, Li J, Wang B, et al Diabetes self-management education reduces risk of all-cause mortality in type 2 diabetes patients: a systematic review and meta-analysis. Endocrine. 2017. 55(3): 712-731.

9. Inzucchi SE, Bergenstal RM, Buse JB, et al Management of hyperglycemia in type 2 diabetes, 2015: a patient-centered approach: update to a position statement of the American Diabetes Association and the European Association for the Study of Diabetes. Diabetes Care. 2015. 38(1): 140-9. 
10. Jia W, Weng J, Zhu D, et al Standards of medical care for type 2 diabetes in China 2019. Diabetes Metab Res Rev. 2019. 35(6): e3158.

11. 6. Glycemic Targets: Standards of Medical Care in Diabetes-2020. Diabetes Care. 2020. 43(Suppl 1): 66-76.

12. Evert AB, Boucher JL, Cypress $M$, et al Nutrition therapy recommendations for the management of adults with diabetes. Diabetes Care. 2014. 37Suppl 1: S120-43.

13. Dyson PA, Twenefour D, Breen C, et al Diabetes UK evidence-based nutrition guidelines for the prevention and management of diabetes. Diabet Med. 2018. 35(5): 541-547.

14. Bolla AM, Caretto A, Laurenzi A, Scavini M, Piemonti L. Low-Carb and Ketogenic Diets in Type 1 and Type 2 Diabetes. Nutrients. 2019. 11(5).

15. Zhang Z, Monro J, Venn BJ. Carbohydrate Knowledge and Expectations of Nutritional Support among Five Ethnic Groups Living in New Zealand with Pre- and Type 2 Diabetes: A Qualitative Study. Nutrients. 2018. 10(9).

16. Raaijmakers LG, Hamers FJ, Martens MK, Bagchus C, de Vries NK, Kremers SP. Perceived facilitators and barriers in diabetes care: a qualitative study among health care professionals in the Netherlands. BMC Fam Pract. 2013. 14: 114.

17. Yin J, Kong AP, Chan JC. Prevention and Care Programs Addressing the Growing Prevalence of Diabetes in China. Curr Diab Rep. 2016. 16(12): 130.

18. WHO, IDF. Definition and diagnosis of diabetes mellitus and intermediate hyperglycaemia: Report of a WHO/IDF consultation. 2006.

19. Jing X, Chen J, Dong Y, et al Related factors of quality of life of type 2 diabetes patients: a systematic review and meta-analysis. Health Qual Life Outcomes. 2018. 16(1): 189.

20. Mokhtari Z, Gheshlagh RG, Kurdi A. Health-related quality of life in Iranian patients with type 2 diabetes: An updated meta-analysis. Diabetes Metab Syndr. 2019. 13(1): 402-407.

21. Buetow S. Thematic analysis and its reconceptualization as 'saliency analysis'. J Health Serv Res Policy. 2010. 15(2): 123-5.

22. Braun V, Clarke V. Using thematic analysis in psychology. Qual Res Psychol. 2006. 3(2): 77-101.

23. Lv Q, Jiang Y, Qi J, et al Using Mobile Apps for Health Management: A New Health Care Mode in China. JMIR Mhealth Uhealth. 2019. 7(6): e10299.

24. Rollo ME, Aguiar EJ, Williams RL, et al eHealth technologies to support nutrition and physical activity behaviors in diabetes self-management. Diabetes Metab Syndr Obes. 2016. 9: 381-390.

25. Schmocker KS, Zwahlen FS, Denecke K. Mobile App for Simplifying Life With Diabetes: Technical Description and Usability Study of GlucoMan. JMIR Diabetes. 2018. 3(1): e6.

26. Cassar AM, Denyer GS, O'Connor HT, Gifford JA. A Qualitative Investigation to Underpin the Development of an Electronic Tool to Assess Nutrition Literacy in Australians Adults. Nutrients. 2018. 10(2).

27. Alidosti M, Sharifirad GR, Golshiri P, Azadbakht L, Hasanzadeh A, Hemati Z. An investigation on the effect of gastric cancer education based on Health Belief Model on knowledge, attitude and nutritional 
practice of housewives. Iran J Nurs Midwifery Res. 2012. 17(4): 256 - 62.

28. Xiong S, Ding M, Li P, Pan S, Li G, He W. A health education model based on knowledge, attitude, and practice used as adjunct therapy for metabolic syndrome complicated with acute pancreatitis: A case report. J Int Med Res. 2020. 48(5): 300060520924272.

29. Frier BM. Hypoglycaemia in diabetes mellitus: epidemiology and clinical implications. Nat Rev Endocrinol. 2014. 10(12): 711 - 22.

30. Umpierrez G, Korytkowski M. Diabetic emergencies - ketoacidosis, hyperglycaemic hyperosmolar state and hypoglycaemia. Nat Rev Endocrinol. 2016. 12(4): 222 - 32.

31. Truman E, Elliott C. Barriers to Food Literacy: A Conceptual Model to Explore Factors Inhibiting Proficiency. J Nutr Educ Behav. 2019. 51(1): 107-111.

32. The Experimental Ecology of Education. Educational Researcher. 1976. 5(9): 5-15.

\section{Figures}

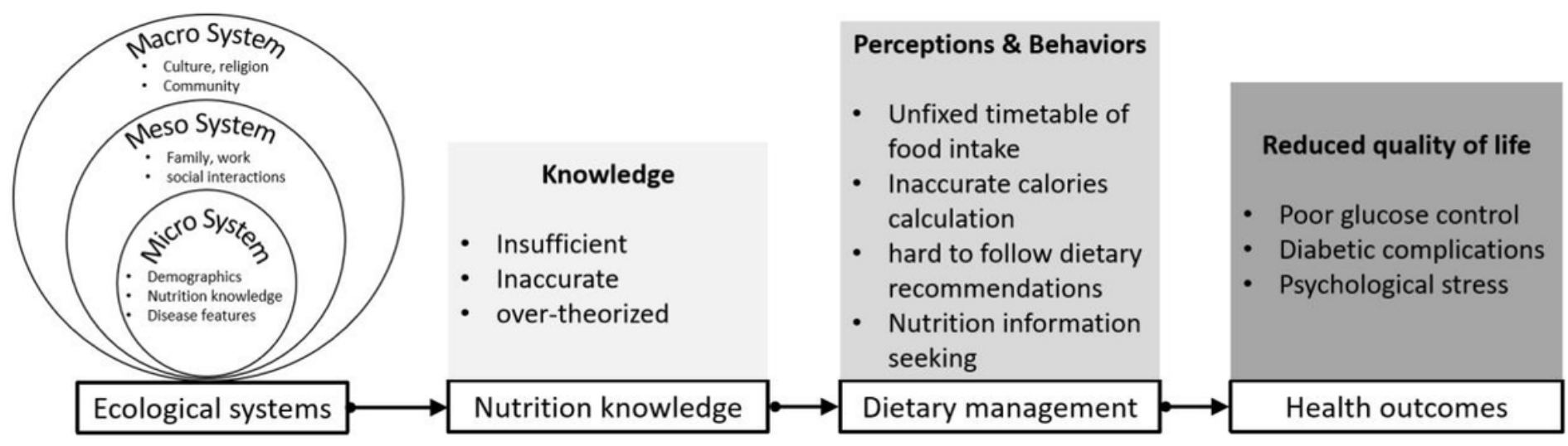

Figure 1

a conceptual model of nutritional problems and its negative impacts on quality of life 